\title{
A Macroscopic Model for an Intermediate State Between Type-I and Type-II Superconductivity
}

\author{
Karel Van Bockstal, Marián Slodička \\ Department of Mathematical Analysis, Research Group $\mathrm{NaM}^{2}$, Ghent University, \\ Galglaan 2, 9000, Ghent, Belgium
}

Received 15 July 2014; accepted 8 December 2014

Published online 9 January 2015 in Wiley Online Library (wileyonlinelibrary.com).

DOI 10.1002/num.21959

\begin{abstract}
A vectorial nonlocal and nonlinear parabolic problem on a bounded domain for an intermediate state between type-I and type-II superconductivity is proposed. The domain is for instance a multiband superconductor that combines the characteristics of both types. The nonlocal term is represented by a (space) convolution with a singular kernel arising in Eringen's model. The nonlinearity is coming from the power law relation by Rhyner. The well-posedness of the problem is discussed under low regularity assumptions and the error estimate for a semi-implicit time-discrete scheme based on backward Euler approximation is established. In the proofs, the monotonicity methods and the Minty-Browder argument are used. (C) 2015 Wiley Periodicals, Inc. Numer Methods Partial Differential Eq 31: 1551-1567, 2015
\end{abstract}

Keywords: error estimates; integro-partial differential equation; nonlinear parabolic equation; nonlocal superconductors; quasi-static Maxwell's equations; singular convolution kernel; time discretization

\section{INTRODUCTION}

In this contribution, a parametrized model is proposed and analyzed for an intermediate state between type-I and type-II superconductivity. It is supposed that a superconducting material occupies a bounded domain $\Omega \subset \mathbb{R}^{3}$ with Lipschitz continuous boundary $\partial \Omega$. The symbol $\boldsymbol{v}$ denotes the outward unit normal vector on $\partial \Omega$. The starting point of the modeling part of this article is the eddy current version of the Maxwell equations (i.e., the displacement current is neglected in Ampère's law):

$$
\begin{array}{ll}
\nabla \times \boldsymbol{H}=\boldsymbol{J}, & \text { Ampère's law }, \\
\nabla \times \boldsymbol{E}=-\partial_{t} \boldsymbol{B}, & \text { Faraday's law }
\end{array}
$$

The eddy current approximation of Maxwell's equations is valid in highly conductive media, see $[1,2]$. A linear dependence of the magnetic induction $\boldsymbol{B}$ on the magnetic field $\boldsymbol{H}$ is

Correspondence to: Karel Van Bockstal, Department of Mathematical Analysis, Research Group NaM2, Ghent University, Galglaan 2, 9000 Ghent, Belgium (e-mail: Karel.VanBockstal@UGent.be)

Contract grant sponsor: Belgian Science Policy (IAP P7/02-project)

(c) 2015 Wiley Periodicals, Inc. 
assumed, namely

$$
\boldsymbol{B}=\mu \boldsymbol{H}
$$

where the constant $\mu>0$ represents the magnetic permeability of the material. Assuming $\left.\nabla \cdot \boldsymbol{B}\right|_{t=0}=0$, applying the divergence operator to Faraday's law (2) and integrating in time ensures that the magnetic induction remains divergence free for any positive time.

Superconductors are usually divided into two main types: type-I and type-II superconductors. The available macroscopic models for both types are pointed out in the following two subsections. For a detailed overview in the literature, see [3-5].

\section{A. Available Macroscopic Models for Type-I Superconductivity}

The starting point for the macroscopic theory for type-I superconductivity is the phenomenological theory of superconductivity in 1935 by London and London. London and London explained that a macroscopic description of type-I superconductors involves a two-fluid model [6,7]. The current density $\boldsymbol{J}$ is supposed to be the sum of a normal and superconducting part, that is

$$
\boldsymbol{J}=\boldsymbol{J}_{\mathrm{n}}+\boldsymbol{J}_{\mathrm{s}}
$$

The normal density current $\boldsymbol{J}_{\mathrm{n}}$ is required to satisfy Ohm's law $\boldsymbol{J}_{\mathrm{n}}=\sigma \boldsymbol{E}$, where $\sigma>0$ is the conductivity of the normal electrons and $\boldsymbol{E}$ the electric field. London and London postulated two equations for $\boldsymbol{J}_{\mathrm{s}}$, in addition to Maxwell's equations, governing the electromagnetic field in a superconductor [6]:

$$
\partial_{t} \boldsymbol{J}_{\mathrm{s}}=\Lambda^{-1} \boldsymbol{E} \quad \text { and } \quad \nabla \times \boldsymbol{J}_{\mathrm{s}}=-\Lambda^{-1} \boldsymbol{B},
$$

where $\Lambda=\frac{m_{e}}{n_{s} e^{2}}$, with $n_{s}$ the number of superelectrons per unit volume, $m_{e}$ and $-e$ the mass and the electric charge of an electron respectively. These equations provide a correct description of two basic properties of superconductors: perfect conductivity and perfect diamagnetism [7]. The second London equation can be rewritten in the local form

$$
J_{\mathrm{s}}=-\Lambda^{-1} \boldsymbol{A},
$$

because there exists a unique magnetic vector potential $\boldsymbol{A}$ since $\boldsymbol{B}$ is divergence free such that $\boldsymbol{B}=\nabla \times \boldsymbol{A}$ and $\nabla \cdot \boldsymbol{A}=0$ if the domain $\Omega$ is simply connected and $\boldsymbol{A} \cdot \boldsymbol{v}=0$ on $\partial \Omega$, cf. [8]. This local theory of London and London is generalized to nonlocal theories, for instance by Pippard and Eringen. Pippard's nonlocal law [9] fails to explain the vanishing of electrical resistance. For this reason, in this contribution, the nonlocal representation of the superconductive current by Eringen [10] is considered. This representation identifies the state of the superconductor, at time $t$, with the field $\boldsymbol{H}(\cdot, t)$ and is given by the linear functional

$$
\boldsymbol{J}_{\mathrm{s}}(\boldsymbol{x}, t)=\int_{\Omega} \sigma_{0}\left(\left|\boldsymbol{x}-\boldsymbol{x}^{\prime}\right|\right)\left(\boldsymbol{x}-\boldsymbol{x}^{\prime}\right) \times \boldsymbol{H}\left(\boldsymbol{x}^{\prime}, t\right) \mathrm{d} \boldsymbol{x}^{\prime}=:-\left(\mathcal{K}_{0} \star \boldsymbol{H}\right)(\boldsymbol{x}, t),(\boldsymbol{x}, t) \in \Omega \times(0, T),
$$

where the singular kernel $\sigma_{0}:(0, \infty) \rightarrow \mathbb{R}$ is defined by

$$
\sigma_{0}(s)= \begin{cases}\frac{\tilde{C}}{2 s^{2}} \exp \left(-\frac{s}{r_{0}}\right) & s<r_{0} \\ 0 & s \geq r_{0},\end{cases}
$$


with $\tilde{C}:=\frac{3}{4 \pi \xi_{0} \Lambda}>0$. The length $\xi_{0}$ is called the coherence length of the material. The points which contribute to the integral are separated by distances of order $r_{0}$ or less, where $r_{0}$ is defined by

$$
r_{0}=\frac{\xi_{0} l}{\xi_{0}+l}
$$

with $l$ the mean free path of the electrons in the material. Moreover, $\sigma_{0}$ is so chosen that it is possible to recover the London equations and the form given by Pippard [7, 10]. Taking the curl of (1) results into the following parabolic integro-differential equation

$$
\mu \partial_{t} \boldsymbol{H}+\sigma^{-1} \nabla \times \nabla \times \boldsymbol{H}+\sigma^{-1} \nabla \times\left(\mathcal{K}_{0} \star \boldsymbol{H}\right)=0 .
$$

The well-posedness under low regularity assumptions of this nonlocal parabolic model is studied into detail in [11]. Also the error estimates for two time-discrete schemes (an implicit and a semi-implicit) based on backward Euler method are derived in [11]. In this contribution, some results of this article are used. Note that a fully discrete approximation scheme is proposed in [12].

\section{B. Available Macroscopic Models for Type-II Superconductivity}

One of the first macroscopic models for type-II superconductors was Bean's critical-state model [13]. The model imposes that a current either flows at the critical level $J_{\mathrm{c}}$ or does not flow at all. Many authors have studied this model [14-19]. Unfortunately, Bean's critical-state model is not fully applicable to superconductors with smooth current-voltage characteristics. Another model frequently used in the modeling of type-II superconductors is the power law constitutive relation by Rhyner [20,21]:

$$
\boldsymbol{E}=\sigma_{\mathrm{c}}^{-n}|\boldsymbol{J}|^{n-1} \boldsymbol{J}, \quad n \in(7,1000),
$$

where $\sigma_{c}$ is some parameter that coordinates the dimensions of both sides in the expression. The value of $n$ depends on the superconducting material and is a measure of the sharpness of the resistive transition. If $n=1$, the relation (6) leads to the linear Ohm's law. If $n \rightarrow \infty$, the solution to the power law formulation converges to the solution to Beans critical-state formulation $[16,17]$. This relation in combination with Maxwell's equations is investigated in [22-26]. Using (1) and taking the curl of (6) leads to the following equation for the magnetic field:

$$
\mu \partial_{t} \boldsymbol{H}+\sigma_{\mathrm{c}}^{-n} \nabla \times\left(|\nabla \times \boldsymbol{H}|^{n-1} \nabla \times \boldsymbol{H}\right)=\mathbf{0} .
$$

\section{Macroscopic Model for an Intermediate State Between Type-I and Type-II Superconductivity}

Recently, there has been increased interest in superconductors with several superconducting components. They arise for instance in multiband superconductors. The classification into types-I and II is insufficient for such multicomponent superconductors [27]. For instance, physicists have found that the material "magnesium diboride" combines the characteristics of both types [28-30]. This leads to a complete new kind of superconductor, the so-called type-1.5 superconductors [29], which allow coexistence of various properties of type-I and type-II superconductors. Type-1.5 materials can be made by placing a thin layer of type-I material onto a thin layer of type-II material 
[31-34]. For more articles about type-1.5 superconductors, see [27, 35, 36]. From this viewpoint, by introducing a real parameter $\beta \geq 1$ and a real function $f(\beta)$, it is proposed to combine Eqs. (5) and (7) to

$$
\begin{gathered}
\mu \partial_{t} \boldsymbol{H}+\sigma^{-1} f(\beta) \nabla \times \nabla \times \boldsymbol{H}+\sigma_{\mathrm{c}}^{-\beta} g(\beta) \nabla \times\left(|\nabla \times \boldsymbol{H}|^{\beta-1} \nabla \times \boldsymbol{H}\right) \\
+\sigma^{-1} f(\beta) \nabla \times\left(\mathcal{K}_{0} \star \boldsymbol{H}\right)=\mathbf{0},
\end{gathered}
$$

with

$$
g(\beta):=1-f(\beta)
$$

and where $f \in C([1, \infty))$ is monotonically decreasing and satisfies $f(1)=1$ and $0 \leq f(\beta) \leq 1$ for $\beta>1$. Moreover, suppose that $f$ is zero or very small for $\beta>7$. For instance, $f$ can take the following form

$$
\begin{aligned}
& f(\beta)= \begin{cases}\frac{(-1)^{\alpha}}{6^{\alpha}}(\beta-7)^{\alpha} & 1 \leq \beta \leq 7 \\
0 & \beta>7,\end{cases} \\
& f(\beta)=\exp (-k \beta),
\end{aligned}
$$

with $\alpha \in \mathbb{N}$ and where $k>0$ gives the speed of convergence to zero. This implies that $g \in C([1, \infty))$ is monotonically increasing with $g(1)=0$ and $0 \leq g(\beta) \leq 1$. Equation (8) simplifies to Eq. (5) for type-I superconductors in the case that $\beta=1$. If $7<\beta<1000$, then Eq. (8) equals or approximates Eq. (7) for type-II superconductivity depending on the choice of $f$. Note that in practical applications $\beta$ is less than 1000 , but in the analysis presented in this article is $\beta$ allowed to be larger. In this contribution, the intermediate phase $(1<\beta \leq 7)$ is attributed to an intermediate state between type-I and type-II superconductivity. The focus in this article is on the mathematical analysis of Eq. (8) and not on its implementation. In future research, the model need to be tested for the different values of $\beta$ and the results should be compared with available results from physics.

The outline of this article is as follows. First, Section II summarizes the mathematical tools. Then, problem (8) is presented into detail in Section III and the well-posedness of the problem is shown in Section IV. More specific, a semi-implicit time-discrete numerical scheme is developed. The existence of a weak solution for each time step is shown. Also the convergence of the method is discussed and error estimates for the time-discretization are derived.

\section{FUNCTIONAL SETTING}

In this section are some standard notations introduced. Let $Q_{T}=\Omega \times(0, T)$ and $\Sigma_{T}=\Gamma \times(0, T)$ for a given final time $T>0$. The Euclidian norm of a vector $\boldsymbol{v}$ in $\mathbb{R}^{3}$ is expressed by $|\boldsymbol{v}|$. The Lebesgue spaces of vector-valued functions with componentwise $p$ th power integrable functions are denoted by $\mathbf{L}^{p}(\Omega)=\left(L^{p}(\Omega)\right)^{3}$ with the usual norm $\|\cdot\|_{p}$. For instance, in the special case $p$ $=2$, the $\mathbf{L}^{2}(\Omega)$ scalar product is denoted by $(\boldsymbol{u}, \boldsymbol{v})=\int_{\Omega} \boldsymbol{u} \cdot \boldsymbol{v} \mathrm{d} \boldsymbol{x}$ and the corresponding norm is $\|\boldsymbol{v}\|=\sqrt{(\boldsymbol{v}, \boldsymbol{v})}$. The analysis of problem (8) take place in a subspace of $\mathbf{H}(\mathbf{c u r l}, \Omega)$. The space $\mathbf{H}(\mathbf{c u r l}, \Omega)$ is a Hilbert space with norm $\|\varphi\|_{\mathbf{H}(\mathbf{c u r l}, \Omega)}^{2}=\|\varphi\|^{2}+\|\nabla \times \varphi\|^{2}$. Consider an abstract Banach space $X$ with norm $\|\cdot\|_{X}$. Then, the spaces $L^{p}((0, T), X)$ and $C([0, T], X)$ consist of functions $u:[0, T] \rightarrow X$ satisfying

$$
\|u\|_{L^{p}((0, T), X)}=\left(\int_{0}^{T}\|u(t)\|_{X}^{p} \mathrm{~d} t\right)^{1 / p}<\infty, \quad\|u\|_{C([0, T], X)}=\max _{[0, T]}\|u(t)\|_{X}<\infty .
$$


Note that $L^{\beta+1}\left((0, T), \mathbf{L}^{\beta+1}(\Omega)\right) \subset L^{2}\left((0, T), \mathbf{L}^{2}(\Omega)\right)$ if $\beta \geq 1$. The space $\operatorname{Lip}\left([0, T], \mathbf{L}^{2}(\Omega)\right)$ is the space of Lipschitz continuous functions $\boldsymbol{F}:[0, T] \rightarrow \mathbf{L}^{2}(\Omega)$. The values $C$, $\varepsilon$, and $C_{\varepsilon}$ are generic and positive constants independent of the discretization parameter $\tau$. The value $\varepsilon$ is small and $C_{\varepsilon}=C+C \varepsilon+C \varepsilon^{-1}$. To reduce the number of arbitrary constants, the notation $a \lesssim b$ is used if there exists a constant $C$ such that $a \leq C b$. Finally, Abel's summation rule is recalled [37]:

$$
2 \sum_{i=1}^{n}\left(a_{i}-a_{i-1}\right) a_{i}=a_{n}^{2}-a_{0}^{2}+\sum_{i=1}^{n}\left(a_{i}-a_{i-1}\right)^{2}, \quad a_{i} \in \mathbb{R} .
$$

\section{A. Important Estimates}

In this section, some useful estimates, who are crucial for the calculations, on the singular kernel and the related convolution appearing in Eq. (8) are mentioned. Using spherical coordinates, one can deduce that $\sigma_{0}(|\boldsymbol{x}|) \boldsymbol{x}$ belongs to $\mathbf{L}^{p}(\Omega)$ for $1 \leq p<3$. This implies that

$$
\left|\boldsymbol{J}_{s}(\boldsymbol{x})\right|=\left|\left(\mathcal{K}_{0} \star \boldsymbol{H}\right)(\boldsymbol{x})\right| \leq C(q)\|\boldsymbol{H}\|_{q}, \quad q>\frac{3}{2}, \quad \forall \boldsymbol{x} \in \Omega .
$$

Therefore, using Young's inequality it is true that

$$
\left(\mathcal{K}_{0} \star \boldsymbol{h}_{1}, \nabla \times \boldsymbol{h}_{2}\right) \leq C_{\varepsilon}\left\|\boldsymbol{h}_{1}\right\|^{2}+\varepsilon\left\|\nabla \times \boldsymbol{h}_{2}\right\|^{2}, \quad \forall \boldsymbol{h}_{1} \in \mathbf{L}^{2}(\Omega), \boldsymbol{h}_{2} \in \mathbf{H}(\operatorname{curl}, \Omega) .
$$

Note that the values $\varepsilon$ and $C_{\varepsilon}$ in the right-hand side (RHS) of this inequality can be switched.

\section{MACROSCOPIC MODEL FOR AN INTERMEDIATE STATE BETWEEN TYPE-I AND TYPE-II SUPERCONDUCTIVITY}

For ease of exposition, it is assumed that $\mu=\sigma=\sigma_{\mathrm{c}}=1$ in (8). Also a possible source term $\boldsymbol{F}$ is considered in the RHS of (8). The aim of this article is to address the well-posedness of the following problem for $\beta \geq 1$ :

$$
\left\{\begin{aligned}
\partial_{t} \boldsymbol{H}+f(\beta) \nabla \times \nabla \times \boldsymbol{H}+g(\beta) \nabla \times\left(|\nabla \times \boldsymbol{H}|^{\beta-1} \nabla \times \boldsymbol{H}\right)+f(\beta) \nabla \times\left(\mathcal{K}_{0} \star \boldsymbol{H}\right)=\boldsymbol{F} & \text { in } Q_{T} \\
\boldsymbol{H} \times \boldsymbol{v}=0 & \text { on } \Sigma_{T} \\
\boldsymbol{H}(\mathrm{x}, 0) & =\boldsymbol{H}_{0} \text { in } \Omega
\end{aligned}\right.
$$

to design a numerical scheme for computations and to derive error estimates for the time discretization. To obtain the magnetic boundary condition in (11), it is assumed that the magnetic field outside the domain $\Omega$ equals zero [8, p. 8].

First, a variational formulation of (11) has to be established. The suitable choice for the space of test functions is

$$
\mathbf{V}_{0}=\left\{\boldsymbol{\varphi} \in \mathbf{L}^{2}(\Omega): \nabla \times \boldsymbol{\varphi} \in \mathbf{L}^{\beta+1}(\Omega) \text { and } \boldsymbol{\varphi} \times \boldsymbol{v}=\mathbf{0} \text { on } \Gamma\right\} \subset \mathbf{H}_{0}(\operatorname{curl}, \Omega) .
$$

This is a closed subspace of the space

$$
\mathbf{V}=\left\{\boldsymbol{\varphi} \in \mathbf{L}^{2}(\Omega): \nabla \times \boldsymbol{\varphi} \in \mathbf{L}^{\beta+1}(\Omega)\right\} \subset \mathbf{H}(\operatorname{curl}, \Omega),
$$


and is endowed with the same graph norm

$$
\|\varphi\|_{\mathbf{V}}=\|\varphi\|_{\mathbf{V}_{0}}=\|\varphi\|_{\mathbf{L}^{2}(\Omega)}+\|\nabla \times \varphi\|_{\mathbf{L}^{\beta+1}(\Omega)} .
$$

Multiplying (11) by any $\varphi \in \mathbf{V}_{0}$, integrating over the domain $\Omega$ and involving the Green theorem, it holds for a.e. $t \in(0, T]$ that

$$
\begin{gathered}
\left(\partial_{t} \boldsymbol{H}(t), \boldsymbol{\varphi}\right)+f(\beta)(\nabla \times \boldsymbol{H}(t), \nabla \times \boldsymbol{\varphi})+g(\beta)\left(|\nabla \times \boldsymbol{H}(t)|^{\beta-1} \nabla \times \boldsymbol{H}(t), \nabla \times \boldsymbol{\varphi}\right) \\
+f(\beta)\left(\mathcal{K}_{0} \star \boldsymbol{H}(t), \nabla \times \boldsymbol{\varphi}\right)=(\boldsymbol{F}(t), \boldsymbol{\varphi}) .
\end{gathered}
$$

For each $t \in(0, T]$, there is looked for a solution $\boldsymbol{H}(t) \in \mathbf{V}_{0}$. Each term of (15) has to be well defined for any $\boldsymbol{H}(t)$ and $\boldsymbol{\varphi} \in \mathbf{V}_{0}$. This can be easily checked using the Cauchy and Hölder's inequality as follows $(\beta \geq 1)$

$$
\begin{aligned}
&\left|\left(\partial_{t} \boldsymbol{H}(t), \boldsymbol{\varphi}\right)\right| \leq\left\|\partial_{t} \boldsymbol{H}(t)\right\|\|\boldsymbol{\varphi}\|, \\
&|(\nabla \times \boldsymbol{H}(t), \nabla \times \boldsymbol{\varphi})| \leq\|\nabla \times \boldsymbol{H}(t)\|\|\nabla \times \boldsymbol{\varphi}\| \lesssim\|\nabla \times \boldsymbol{H}(t)\|_{\mathbf{L}^{\beta+1}(\Omega)}\|\nabla \times \boldsymbol{\varphi}\|_{\mathbf{L}^{\beta+1}(\Omega)}, \\
&\left|\left(|\nabla \times \boldsymbol{H}(t)|^{\beta-1} \nabla \times \boldsymbol{H}(t), \nabla \times \boldsymbol{\varphi}\right)\right| \leq \int_{\Omega}|\nabla \times \boldsymbol{H}(t)|^{\beta}|\nabla \times \boldsymbol{\varphi}| \\
& \leq\|\nabla \times \boldsymbol{H}(t)\|_{\mathbf{L}^{\beta+1}(\Omega)}^{\frac{\beta}{\beta+1}}\|\nabla \times \boldsymbol{\varphi}\|_{\mathbf{L}^{\beta+1}(\Omega)}^{\frac{1}{\beta+1}}, \\
&\left|\left(\mathcal{K}_{0} \star \boldsymbol{H}(t), \nabla \times \boldsymbol{\varphi}\right)\right| \stackrel{(10)}{\lesssim}\|\boldsymbol{H}(t)\|^{2}+\|\nabla \times \boldsymbol{\varphi}\|^{2} \lesssim\|\boldsymbol{H}(t)\|^{2}+\|\nabla \times \boldsymbol{\varphi}\|_{\mathbf{L}^{\beta+1}(\Omega)}^{2}, \\
&|(\boldsymbol{F}(t), \boldsymbol{\varphi})| \leq\|\boldsymbol{F}(t)\|\|\boldsymbol{\varphi}\| .
\end{aligned}
$$

Now, it is possible to define the following weak formulation.

Definition 3.1. Let $\beta \geq 1, \boldsymbol{H}_{0} \in \mathbf{V}$ and $\boldsymbol{F} \in L^{2}\left((0, T), \mathbf{L}^{2}(\Omega)\right)$. The variational formulation of (11) reads as: find $\boldsymbol{H} \in C\left([0, T], \mathbf{L}^{2}(\Omega)\right)$ with $\nabla \times \boldsymbol{H} \in L^{\beta+1}\left((0, T), \mathbf{L}^{\beta+1}(\Omega)\right)$ and $\partial_{t} \boldsymbol{H} \in L^{2}\left([0, T], \mathbf{L}^{2}(\Omega)\right)$ such that

$$
\begin{gathered}
\left(\partial_{t} \boldsymbol{H}(t), \boldsymbol{\varphi}\right)+f(\beta)(\nabla \times \boldsymbol{H}(t), \nabla \times \boldsymbol{\varphi})+g(\beta)\left(|\nabla \times \boldsymbol{H}(t)|^{\beta-1} \nabla \times \boldsymbol{H}(t), \nabla \times \boldsymbol{\varphi}\right) \\
+f(\beta)\left(\mathcal{K}_{0} \star \boldsymbol{H}(t), \nabla \times \boldsymbol{\varphi}\right)=(\boldsymbol{F}(t), \boldsymbol{\varphi}), \quad \forall \boldsymbol{\varphi} \in \mathbf{V}_{0},
\end{gathered}
$$

for a.e. $t \in[0, T]$.

The dual space of $\mathbf{V}_{0}$ is denoted by $\mathbf{V}_{0}^{*}$. The following lemma states the reflexivity of the spaces $\mathbf{V}$ and $\mathbf{V}_{0}$.

Lemma 1. The vector spaces $V$ and $\mathbf{V}_{0}$ are reflexive Banach spaces.

Proof. The proof can be done in an analogous way of the proof of [38, Lemma 1].

The following technical lemma is crucial in the proofs. The interested reader is referred to [39, p. 13] for the proof. 
Lemma 2. Let $\alpha \geq 1$. There exists a positive constant $C_{0}(\alpha)=\frac{1}{4 \cdot 12^{\frac{\alpha+1}{2}}}$ such that for any $\boldsymbol{H}_{1}, \boldsymbol{H}_{2} \in \mathbf{V}$ hold

$$
\begin{aligned}
& \left(\left|\nabla \times \boldsymbol{H}_{1}\right|^{\alpha-1} \nabla \times \boldsymbol{H}_{1}-\left|\nabla \times \boldsymbol{H}_{2}\right|^{\alpha-1} \nabla \times \boldsymbol{H}_{2}, \nabla \times\left(\boldsymbol{H}_{1}-\boldsymbol{H}_{2}\right)\right) \\
& \geq C_{0}(\alpha)\left\|\nabla \times\left(\boldsymbol{H}_{1}-\boldsymbol{H}_{2}\right)\right\|_{\mathbf{L}^{\alpha+1}(\Omega)}^{\alpha+1} .
\end{aligned}
$$

Following theorem guarantees the uniqueness of the solution to problem (11).

Theorem 1 (Uniqueness). The problem (11) admits at most one solution $\partial_{t} \boldsymbol{H} \in L^{2}([0, T]$, $\left.\mathbf{L}^{2}(\Omega)\right)$ with $\nabla \times \boldsymbol{H} \in L^{\beta+1}\left((0, T), \mathbf{L}^{\beta+1}(\Omega)\right)$ if $\boldsymbol{H}_{0} \in \mathbf{L}^{2}(\Omega)$.

Proof. Assume that we have two solutions $\boldsymbol{H}_{1}$ and $\boldsymbol{H}_{2}$. Set $\boldsymbol{H}=\boldsymbol{H}_{1}-\boldsymbol{H}_{2}$. Then $\boldsymbol{H}_{0}=0$. Subtract Eq. (16) for $\boldsymbol{H}=\boldsymbol{H}_{1}$ from (16) for $\boldsymbol{H}=\boldsymbol{H}_{2}$. Setting $\boldsymbol{\varphi}=\boldsymbol{H}$ into the resulting equation and integrating in time for $t \in(0, T)$, we find thanks to Lemma 2 that

$$
\frac{1}{2}\|\boldsymbol{H}(t)\|^{2}+f(\beta) \int_{0}^{t}\|\nabla \times \boldsymbol{H}\|^{2}+g(\beta) C_{0} \int_{0}^{t}\|\nabla \times \boldsymbol{H}\|_{\mathbf{L}^{\beta+1}(\Omega)}^{\beta+1} \leq-f(\beta) \int_{0}^{t}\left(\mathcal{K}_{0} \star \boldsymbol{H}, \nabla \times \boldsymbol{H}\right) .
$$

Using inequality (10) for the term on the RHS, we arrive at

$$
\|\boldsymbol{H}(t)\|^{2}+f(\beta) \int_{0}^{t}\|\nabla \times \boldsymbol{H}\|^{2}+g(\beta) C_{0} \int_{0}^{t}\|\nabla \times \boldsymbol{H}\|_{\mathbf{L}^{\beta+1}(\Omega)}^{\beta+1} \leq C_{\varepsilon} \int_{0}^{t}\|\boldsymbol{H}\|^{2}+\varepsilon \int_{0}^{t}\|\nabla \times \boldsymbol{H}\|^{2} .
$$

We consider four cases:

- $\beta=1$ : then $f(\beta)=1$ and $g(\beta)=0$. Fixing a sufficiently small positive $\varepsilon$ and applying the Grönwall argument, we get that $\boldsymbol{H}=0$ a.e. in $Q_{T}$;

- $1<\beta<7$ : then $f$ and $g$ are strict positive. Again fixing a sufficiently small positive $\varepsilon$ and applying the Grönwall argument gives that $\boldsymbol{H}=0$ a.e. in $Q_{T}$;

- $\beta \geq 7$ and $f(\beta)=0$ for $\beta \geq 7$ : thus $g(\beta)=\sigma_{c}^{-\beta}$ and the convolution term disappears out of the problem. We immediately obtain that $\boldsymbol{H}=0$ a.e. in $Q_{T}$;

- $\beta \geq 7$ and $f(\beta)>0$ for $\beta \geq 7$ but very small: analogously as the case $1<\beta<7$.

Remark 1. In the previous theorem are four cases considered depending on the value of the parameter $\beta$. These situations are not repeated in the remainder of the article but should be reconsidered by the reader in the stability analysis and the a priori estimates.

The following theorem describes the natural stability of the solution $\boldsymbol{H}$ of (11). This will give insight into the spaces where we look for a solution.

Theorem 2 (Stability). Suppose $\boldsymbol{F} \in L^{2}\left((0, T), \mathbf{L}^{2}(\Omega)\right)$.

1. If $\boldsymbol{H}_{0} \in \mathbf{L}^{2}(\Omega)$ then

$$
\max _{t \in[0, T]}\|\boldsymbol{H}(t)\|^{2}+\int_{0}^{T}\|\nabla \times \boldsymbol{H}\|_{\mathbf{L}^{\beta+1}(\Omega)}^{\beta+1} \leq C .
$$

2. If $\nabla \cdot \boldsymbol{F}(t)=0=\nabla \cdot \boldsymbol{H}_{0}$ then $\nabla \cdot \boldsymbol{H}(t)=0$ for any $t \in[0, T]$. 
3. If $\boldsymbol{H}_{0} \in \mathbf{V}$ then

$$
\max _{t \in[0, T]}\|\nabla \times \boldsymbol{H}(t)\|_{\mathbf{L}^{\beta+1}(\Omega)}^{\beta+1}+\int_{0}^{T}\left\|\partial_{t} \boldsymbol{H}\right\|^{2} \leq C .
$$

4. If $\partial_{t} \boldsymbol{F} \in L^{2}\left((0, T), \mathbf{L}^{2}(\Omega)\right), \nabla \times\left(\mathcal{K}_{0} \star \boldsymbol{H}_{0}\right) \in \mathbf{L}^{2}(\Omega), \boldsymbol{H}_{0} \in \mathbf{V}_{0}, \nabla \times \nabla \times \boldsymbol{H}_{0} \in \mathbf{L}^{2}(\Omega)$ and

5. $\nabla \times\left[\left|\nabla \times \boldsymbol{H}_{0}\right|^{\beta-1} \nabla \times \boldsymbol{H}_{0}\right] \in \mathbf{L}^{2}(\Omega)$ then

$$
\max _{t \in[0, T]}\left\|\partial_{t} \boldsymbol{H}(t)\right\|^{2} \leq C
$$

Proof. i. Setting $\boldsymbol{\varphi}=\boldsymbol{H}(t)$ in (16) and integrating in time for $t \in(0, T)$, we get due to Lemma 2 that

$$
\begin{aligned}
& \frac{\|\boldsymbol{H}(t)\|^{2}}{2}+f(\beta) \int_{0}^{t}\|\nabla \times \boldsymbol{H}\|^{2}+g(\beta) \int_{0}^{T}\|\nabla \times \boldsymbol{H}\|_{\mathbf{L}^{\beta+1}(\Omega)}^{\beta+1} \leq \frac{\left\|\boldsymbol{H}_{0}\right\|^{2}}{2} \\
& +\int_{0}^{t}(\boldsymbol{F}, \boldsymbol{H})-f(\beta) \int_{0}^{t}\left(\mathcal{K}_{0} \star \boldsymbol{H}, \nabla \times \boldsymbol{H}\right) .
\end{aligned}
$$

Using the Cauchy and Young inequalities, inequality (10) for the last term on the RHS and Grönwall's argument (depending on the value of $\beta$ ) conclude the proof.

ii. Take the divergence of (11) or set $\varphi=\nabla \phi$ with $\phi \in C_{0}^{\infty}(\Omega)$ in (16). Then, integrate in time to arrive at $\nabla \cdot \boldsymbol{H}(t)=\nabla \cdot \boldsymbol{H}_{0}=0$ for all $t \in[0, T]$.

iii. Note that $|\mathbf{u}|^{\beta-1} \mathbf{u} \cdot \partial_{t} \mathbf{u}=\partial_{t} \frac{|\mathbf{u}|^{\beta+1}}{\beta+1}$. Now, we set $\boldsymbol{\varphi}=\partial_{t} \boldsymbol{H}$ in (16) and integrate in time for $t \in(0, T)$ to obtain

$$
\begin{aligned}
\int_{0}^{t} & \left\|\partial_{t} \boldsymbol{H}\right\|^{2}+\frac{f(\beta)}{2}\|\nabla \times \boldsymbol{H}(t)\|^{2}+\frac{g(\beta)}{\beta+1}\|\nabla \times \boldsymbol{H}(t)\|_{\mathbf{L}^{\beta+1}(\Omega)}^{\beta+1} \\
= & \frac{f(\beta)}{2}\left\|\nabla \times \boldsymbol{H}_{0}\right\|^{2}+\frac{g(\beta)}{\beta+1}\left\|\nabla \times \boldsymbol{H}_{0}\right\|_{\mathbf{L}^{\beta+1}(\Omega)}^{\beta+1}+\int_{0}^{t}\left(\boldsymbol{F}, \partial_{t} \boldsymbol{H}\right) \\
& \quad-f(\beta) \int_{0}^{t}\left(\mathcal{K}_{0} \star \boldsymbol{H}, \nabla \times \partial_{t} \boldsymbol{H}\right)
\end{aligned}
$$

The last term in the RHS can be estimated as in [11, Theorem 2 (iii)] using integration by parts. Afterward, the result follows straight.

iv. We differentiate (16) with respect to the time variable. Therefore, we need that (16) is fulfilled for $t=0$. Knowing that

$$
\begin{aligned}
\nabla \times \nabla \times \boldsymbol{H}_{0} & \in \mathbf{L}^{2}(\Omega), & & \nabla \times\left[\left|\nabla \times \boldsymbol{H}_{0}\right|^{\beta-1} \nabla \times \boldsymbol{H}_{0}\right] \in \mathbf{L}^{2}(\Omega), \\
\nabla \times\left(\mathcal{K}_{0} \star \boldsymbol{H}_{0}\right) & \in \mathbf{L}^{2}(\Omega), & & \boldsymbol{H}_{0} \in \mathbf{V}_{0},
\end{aligned}
$$

we may define

$$
\begin{aligned}
\partial_{t} \boldsymbol{H}(0)= & \boldsymbol{F}(0)-f(\beta) \nabla \times \nabla \times \boldsymbol{H}_{0}-g(\beta) \nabla \times\left(\left|\nabla \times \boldsymbol{H}_{0}\right|^{\beta-1} \nabla \times \boldsymbol{H}_{0}\right) \\
& -f(\beta) \nabla \times\left(\mathcal{K}_{0} \star \boldsymbol{H}_{0}\right),
\end{aligned}
$$


that is,

$$
\left\|\partial_{t} \boldsymbol{H}(0)\right\| \lesssim 1
$$

Now, we set $\boldsymbol{\varphi}=\partial_{t} \boldsymbol{H}$ and integrate in time for $t \in(0, T)$ to get that

$$
\begin{aligned}
\frac{1}{2}\left\|\partial_{t} \boldsymbol{H}(t)\right\|^{2}+f(\beta) \int_{0}^{t}\left\|\nabla \times \partial_{t} \boldsymbol{H}\right\|^{2} \\
\quad+g(\beta) \int_{0}^{t} \int_{\Omega}|\nabla \times \boldsymbol{H}|^{\beta-1}\left[\left|\nabla \times \partial_{t} \boldsymbol{H}\right|^{2}+(\beta-1)\left(\partial_{t}|\nabla \times \boldsymbol{H}|\right)^{2}\right] \\
=\frac{1}{2}\left\|\partial_{t} \boldsymbol{H}(0)\right\|^{2}+\int_{0}^{t}\left(\partial_{t} \boldsymbol{F}, \partial_{t} \boldsymbol{H}\right)-f(\beta) \int_{0}^{t}\left(\mathcal{K}_{0} \star \partial_{t} \boldsymbol{H}, \nabla \times \partial_{t} \boldsymbol{H}\right) .
\end{aligned}
$$

In the last step, we have used that $\partial_{t}\left(|\mathbf{u}|^{\alpha-1} \mathbf{u}\right) \cdot \partial_{t} \mathbf{u}=\left[\left|\partial_{t} \mathbf{u}\right|^{2}+(\alpha-1)\left(\partial_{t}|\mathbf{u}|\right)^{2}\right]|\mathbf{u}|^{\alpha-1}$. Using the Cauchy and Young inequalities, (iii) and (10) to the RHS, and applying the Grönwall argument (depending on the value of $\beta$ ), we arrive at the result. Note that the second and third term in the left-hand side (LHS) cannot be zero together.

Remark 2. To obtain higher regularity of the solution, higher regularity of the known data is required, see Lemma 2(iv).

\section{EXISTENCE OF A SOLUTION}

To address the existence of a solution to (11), the semidiscretization in time is used. This discretization is based on Rothe's method [40]. The interval $[0, T]$ is divided into $n$ equidistant subintervals $\left[t_{i-1}, t_{i}\right]$ with time step $\tau=\frac{T}{n}<1$, thus $t_{i}=i \tau, i=0, \ldots, n$. With the standard notation for the discretized fields

$$
\boldsymbol{h}_{i} \approx \boldsymbol{H}\left(t_{i}\right) \quad \text { and } \quad \delta \boldsymbol{h}_{i}=\frac{\boldsymbol{h}_{i}-\boldsymbol{h}_{i-1}}{\tau}
$$

the following linear recurrent semi-implicit scheme is proposed to approximate the original problem

$$
\left\{\begin{aligned}
\left(\delta \boldsymbol{h}_{i}, \boldsymbol{\varphi}\right)+f(\beta)\left(\nabla \times \boldsymbol{h}_{i}, \nabla \times \boldsymbol{\varphi}\right) & \\
+g(\beta)\left(\left|\nabla \times \boldsymbol{h}_{i}\right|^{\beta-1} \nabla \times \boldsymbol{h}_{i}, \nabla \times \boldsymbol{\varphi}\right) & =\left(\boldsymbol{f}_{i}, \boldsymbol{\varphi}\right)-f(\beta)\left(\mathcal{K}_{0} \star \boldsymbol{h}_{i-1}, \nabla \times \boldsymbol{\varphi}\right) ; \\
\boldsymbol{h}_{0} & =\boldsymbol{H}_{0}
\end{aligned}\right.
$$

which is equivalent to solving on each time step the operator equation $A(\mathbf{u})=\boldsymbol{f}_{i}^{*}$ where $A: \mathbf{V}_{0} \rightarrow \mathbf{V}_{0}^{*}$ is defined by

$$
\langle A(\mathbf{u}), \mathbf{v}\rangle=\left(\frac{\mathbf{u}}{\tau}, \mathbf{v}\right)+f(\beta)(\nabla \times \mathbf{u}, \nabla \times v)+g(\beta)\left(|\nabla \times \mathbf{u}|^{\beta-1} \nabla \times \mathbf{u}, \nabla \times \mathbf{v}\right)
$$

and $\boldsymbol{f}_{i}^{*}: \mathbf{V}_{0} \rightarrow \mathbb{R}$ by

$$
\left\langle\boldsymbol{f}_{i}^{*}, \mathbf{v}\right\rangle=\left(\boldsymbol{f}_{i}, \mathbf{v}\right)-f(\beta)\left(\mathcal{K}_{0} \star \boldsymbol{h}_{i-1}, \nabla \times \mathbf{v}\right)+\left(\frac{\boldsymbol{h}_{i-1}}{\tau}, \mathbf{v}\right) .
$$


The solution from the previous time step is evaluated into the convolution term instead of the solution of the actual time step because this should be easier to implement. As mentioned before, the focus in this contribution is not on the implementation of the numerical scheme, but on its analysis. The existence and uniqueness of a weak solution on each time step is guaranteed by the following theorem.

Theorem 3 (Uniqueness on a single time step). Assume $\boldsymbol{H}_{0} \in \mathbf{L}^{2}(\Omega)$ and $\boldsymbol{F} \in L^{2}((0, T)$, $\mathbf{L}^{2}(\Omega)$ ). Then, there exists a $\tau_{0}>0$ such that the variational problem (17) has a unique solution for any $i=1, \ldots, n$ and any $\tau<\tau_{0}$.

Proof. Note that the space $\mathbf{V}_{0}^{*}$ is a reflexive Banach space, see Lemma 1. Therefore, following the theory of monotone operators [41, Theorem 18.2, Remark 18.2], the operator equation $A(\mathbf{u})=f_{i}^{*}$ has a unique solution on each time step because $A$ is a strictly monotone, coercive, hemicontinuous operator and $\boldsymbol{f}_{i}^{*} \in \mathbf{V}_{0}^{*}$ starting from $\boldsymbol{H}_{0} \in \mathbf{L}^{2}(\Omega)$. In particular, the strict monotonicity of $A$ follows from Lemma 2.

First, basic stability result for $\mathrm{h}_{i}$ are derived. The a priori estimates in part (i) and (iii) in the following theorem serve as uniform bounds to prove convergence. In the proofs, the following lemma is needed, see [42, Lemma 2.3].

Lemma 3. Let $g: \mathbb{R} \rightarrow \mathbb{R}$ be a nonnegative continuous function such that $G(s):=g(s) s$ is monotonically increasing. Let $\Phi_{G}$ be a primitive function of $G$. Then for any $\mathbf{x}, \mathbf{y} \in \mathbb{R}^{3}$ it holds that

$$
\Phi_{G}(|\mathbf{y}|)-\Phi_{G}(|\mathbf{x}|) \leq g(|\mathbf{y}|) \mathbf{y} \cdot(\mathbf{y}-\mathbf{x})
$$

Lemma 4 (A priori estimates). Suppose that $\boldsymbol{F} \in L^{2}\left((0, T), \mathbf{L}^{2}(\Omega)\right)$.

i. Let $\boldsymbol{H}_{0} \in \mathbf{L}^{2}(\Omega)$. Then, there exists a positive constant $C$ such that

$$
\max _{1 \leq i \leq n}\left\|\boldsymbol{h}_{i}\right\|^{2}+\sum_{i=1}^{n}\left\|\boldsymbol{h}_{i}-\boldsymbol{h}_{i-1}\right\|^{2}+\sum_{i=1}^{n}\left\|\nabla \times \boldsymbol{h}_{i}\right\|_{\mathbf{L}^{\beta+1}(\Omega)}^{\beta+1} \tau \leq C
$$

for all $\tau<\tau_{0}$.

ii. If $\nabla \cdot \boldsymbol{H}_{0}=0=\nabla \cdot \boldsymbol{f}_{i}$ then $\nabla \cdot \boldsymbol{h}_{i}=0$ for all $i=1, \ldots, n$.

iii. If $\boldsymbol{H}_{0} \in \mathbf{V}$ then

$$
\max _{1 \leq i \leq n}\left\|\nabla \times \boldsymbol{h}_{i}\right\|_{\mathbf{L}^{\beta+1}(\Omega)}^{\beta+1}+\sum_{i=1}^{n}\left\|\delta \boldsymbol{h}_{i}\right\|^{2} \tau \leq C
$$

for all $\tau<\tau_{0}$.

Proof. i. Setting $\boldsymbol{\varphi}=\boldsymbol{h}_{i}$ in (17), multiplying by $\tau$ and summing up for $i=1, \ldots, j$, we have

$$
\sum_{i=1}^{j}\left(\delta \boldsymbol{h}_{i}, \boldsymbol{h}_{i}\right) \tau+f(\beta) \sum_{i=1}^{j}\left\|\nabla \times \boldsymbol{h}_{i}\right\|^{2} \tau+g(\beta) \sum_{i=1}^{j}\left(\left|\nabla \times \boldsymbol{h}_{i}\right|^{\beta-1} \nabla \times \boldsymbol{h}_{i}, \nabla \times \boldsymbol{h}_{i}\right) \tau
$$




$$
=\sum_{i=1}^{j}\left(\boldsymbol{f}_{i}, \boldsymbol{h}_{i}\right) \tau-f(\beta) \sum_{i=1}^{j}\left(\mathcal{K}_{0} \star \boldsymbol{h}_{i-1}, \nabla \times \boldsymbol{h}_{i}\right) \tau
$$

The first term on the LHS can be rewritten using Abel's summation rule. The third term can be estimated below thanks to Lemma 2. The second term on the RHS can be estimated as in [11, Lemma 2(i)]. An application of Grönwall's lemma completes the proof.

ii. The result can be readily obtained by applying the divergence operator to

$$
\delta \boldsymbol{h}_{i}+f(\beta) \nabla \times \nabla \times \boldsymbol{h}_{i}+g(\beta) \nabla \times\left(\left|\nabla \times \boldsymbol{h}_{i}\right|^{\beta-1} \nabla \times \boldsymbol{h}_{i}\right)+f(\beta) \nabla \times\left(\mathcal{K}_{0} \star \boldsymbol{h}_{i-1}\right)=\boldsymbol{f}_{i},
$$

or setting $\varphi=\nabla \phi$ with $\phi \in C_{0}^{\infty}(\Omega)$ in (17).

iii. Setting $\boldsymbol{\varphi}=\delta \boldsymbol{h}_{i}$ in (17), multiplying by $\tau$ and summing up for $i=1, \ldots, j$, we have

$$
\begin{aligned}
& \sum_{i=1}^{j}\left\|\delta \boldsymbol{h}_{i}\right\|^{2} \tau+f(\beta) \sum_{i=1}^{j}\left(\nabla \times \boldsymbol{h}_{i}, \nabla \times \boldsymbol{h}_{i}-\nabla \times \boldsymbol{h}_{i-1}\right) \\
& \quad+g(\beta) \sum_{i=1}^{j}\left(\left|\nabla \times \boldsymbol{h}_{i}\right|^{\beta-1} \nabla \times \boldsymbol{h}_{i}, \nabla \times \delta \boldsymbol{h}_{i}\right) \tau=\sum_{i=1}^{j}\left(\boldsymbol{f}_{i}, \delta \boldsymbol{h}_{i}\right) \tau \\
& \quad-f(\beta) \sum_{i=1}^{j}\left(\mathcal{K}_{0} \star \boldsymbol{h}_{i-1}, \nabla \times \delta \boldsymbol{h}_{i}\right) \tau .
\end{aligned}
$$

The second term in the LHS can be estimated by Abel's summation rule. The third term on the LHS can be estimated below using Lemma 3 with $g(s)=s^{\beta-1}$. We obtain

$$
\begin{aligned}
\sum_{i=1}^{j}\left(\left|\nabla \times \boldsymbol{h}_{i}\right|^{\beta-1} \nabla \times \boldsymbol{h}_{i}, \nabla \times \delta \boldsymbol{h}_{i}\right) \tau & \geq \frac{1}{\beta+1} \sum_{i=1}^{j} \int_{\Omega}\left[\left|\nabla \times \boldsymbol{h}_{i}\right|^{\beta+1}-\left|\nabla \times \boldsymbol{h}_{i-1}\right|^{\beta+1}\right] \\
& =\frac{1}{\beta+1}\left(\left\|\nabla \times \boldsymbol{h}_{j}\right\|_{\mathbf{L}^{\beta+1}(\Omega)}^{\beta+1}-\left\|\nabla \times \boldsymbol{H}_{0}\right\|_{\mathbf{L}^{\beta+1}(\Omega)}^{\beta+1}\right) .
\end{aligned}
$$

The last term on the RHS can be estimated as in [11, Lemma 2(iii)]. Using (i), we conclude the proof.

The existence of a weak solution is proved using Rothe's method. The following piecewise linear in time vector fields $\boldsymbol{H}_{n}$

$$
\begin{aligned}
& \boldsymbol{H}_{n}(0)=\boldsymbol{H}_{0} \\
& \boldsymbol{H}_{n}(t)=\boldsymbol{h}_{i-1}+\left(t-t_{i-1}\right) \delta \boldsymbol{h}_{i} \quad \text { for } t \in\left(t_{i-1}, t_{i}\right], \quad i=1, \ldots, n
\end{aligned}
$$

and the piecewise constant in time fields $\overline{\boldsymbol{H}}_{n}$ are introduced

$$
\overline{\boldsymbol{H}}_{n}(0)=\boldsymbol{H}_{0}, \quad \overline{\boldsymbol{H}}_{n}(t)=\boldsymbol{h}_{i}, \quad \text { for } t \in\left(t_{i-1}, t_{i}\right], \quad i=1, \ldots, n .
$$

Similarly, the vector field $\overline{\boldsymbol{F}}_{n}$ is defined. The variational formulation (17) can be rewritten as

$$
\begin{aligned}
& \left(\partial_{t} \boldsymbol{H}_{n}(t), \boldsymbol{\varphi}\right)+f(\beta)\left(\nabla \times \overline{\boldsymbol{H}}_{n}(t), \nabla \times \boldsymbol{\varphi}\right)+g(\beta)\left(\left|\nabla \times \overline{\boldsymbol{H}}_{n}(t)\right|^{\beta-1} \nabla \times \overline{\boldsymbol{H}}_{n}(t), \nabla \times \boldsymbol{\varphi}\right) \\
& \quad=\left(\overline{\boldsymbol{F}}_{n}(t), \boldsymbol{\varphi}\right)-f(\beta)\left(\mathcal{K}_{0} \star \overline{\boldsymbol{H}}_{n}(t-\tau), \nabla \times \boldsymbol{\varphi}\right) .
\end{aligned}
$$


Now, the convergence of the sequences $\boldsymbol{H}_{n}$ and $\overline{\boldsymbol{H}}_{n}$ to the unique weak solution of (11) is proved if $\tau \rightarrow 0$ or $n \rightarrow \infty$.

Theorem 4 (Existence). Let $\boldsymbol{H}_{0} \in \mathbf{V}$ and $\boldsymbol{F} \in L^{2}\left((0, T), \mathbf{L}^{2}(\Omega)\right)$. Assume that $\nabla \cdot \boldsymbol{H}_{0}=0=$ $\nabla \cdot \boldsymbol{f}(t)$ for any time $t \in[0, T]$. Then, there exists a weak solution $H$ such that

i. $\overline{\boldsymbol{H}}_{n} \rightarrow \boldsymbol{H}$ in $L^{2}\left((0, T), \mathbf{L}^{2}(\Omega)\right), \nabla \times \overline{\boldsymbol{H}}_{n} \rightarrow \nabla \times \boldsymbol{H}$ in $L^{\beta+1}\left((0, T), \mathbf{L}^{\beta+1}(\Omega)\right)$ and $\boldsymbol{H}_{n} \rightarrow \boldsymbol{H}$ in $L^{2}\left((0, T), \mathbf{L}^{2}(\Omega)\right)$;

ii. $\boldsymbol{H}_{n} \rightarrow \boldsymbol{H}$ in $C\left([0, T], \mathbf{L}^{2}(\Omega)\right), \partial_{t} \boldsymbol{H}_{n} \rightarrow \partial_{t} \boldsymbol{H}$ in $L^{2}\left((0, T), \mathbf{L}^{2}(\Omega)\right)$ and $\overline{\boldsymbol{H}}_{n} \rightarrow \boldsymbol{H}$ in $L^{2}\left([0, T], \mathbf{L}^{2}(\Omega)\right)$;

iii. $\left|\nabla \times \overline{\boldsymbol{H}}_{n}\right|^{\beta-1} \nabla \times \overline{\boldsymbol{H}}_{n} \rightarrow|\nabla \times \boldsymbol{H}|^{\beta-1} \nabla \times \boldsymbol{H}$ in $L^{\frac{\beta+1}{\beta}}\left((0, T), \mathbf{L}{ }^{\frac{\beta+1}{\beta}}(\Omega)\right)$

iv. $\boldsymbol{H} \in C\left([0, T], \mathbf{L}^{2}(\Omega)\right)$ is a weak solution of $(16)$.

Proof. $\quad$ i. The spaces $L^{2}\left((0, T), \mathbf{L}^{2}(\Omega)\right)$ and $L^{\beta+1}\left((0, T), \mathbf{L}^{\beta+1}(\Omega)\right)$ are reflexive Banach spaces. Thanks to Lemma 4(i) and (iii), the sequence $\overline{\boldsymbol{H}}_{n}$ is bounded in $L^{2}\left((0, T), \mathbf{L}^{2}(\Omega)\right)$, the sequence $\nabla \times \overline{\boldsymbol{H}}_{n}$ is bounded in $L^{\beta+1}\left((0, T), \mathbf{L}^{\beta+1}(\Omega)\right)$ and the sequence $\boldsymbol{H}_{n}$ is bounded in $L^{2}\left((0, T), \mathbf{L}^{2}(\Omega)\right)$. Therefore, the sequence $\overline{\boldsymbol{H}}_{n}$ contains a weakly convergence subsequence (denoted by the same symbol again) such that $\overline{\boldsymbol{H}}_{n} \rightarrow \boldsymbol{H}$ in $L^{2}\left((0, T), \mathbf{L}^{2}(\Omega)\right)$ and $\nabla \times \overline{\boldsymbol{H}}_{n} \rightarrow \mathbf{z}$ in $L^{\beta+1}\left((0, T), \mathbf{L}^{\beta+1}(\Omega)\right)$ [43, Theorem 1 p. 126]. According to the Hahn-Banach theorem, it is easy to show that $\mathbf{z}=\nabla \times \boldsymbol{H} \in L^{\beta+1}\left((0, T), \mathbf{L}^{\beta+1}(\Omega)\right)$. Using Lemma 4(i) gives

$$
\lim _{n \rightarrow \infty}\left\|\boldsymbol{H}_{n}-\overline{\boldsymbol{H}}_{n}\right\|_{L^{2}\left((0, T), \mathbf{L}^{2}(\Omega)\right)}^{2}=0
$$

Thus, $\overline{\boldsymbol{H}}_{n}$ and $\boldsymbol{H}_{n}$ have the same limit in $L^{2}\left((0, T), \mathbf{L}^{2}(\Omega)\right)$. Therefore, $\boldsymbol{H}_{n} \rightarrow \boldsymbol{H}$ in $L^{2}\left((0, T), \mathbf{L}^{2}(\Omega)\right)$.

ii. Lemma 4 implies that

$$
\boldsymbol{h}_{i} \in \mathbf{L}^{2}(\Omega), \quad \nabla \times \boldsymbol{h}_{i} \in \mathbf{L}^{2}(\Omega), \quad \nabla \cdot \boldsymbol{h}_{i}=0 \text { in } \Omega, \quad \boldsymbol{h}_{i} \times \boldsymbol{v}=\mathbf{0} \text { on } \Gamma, \quad i=1, \ldots, n .
$$

Using [8, Theorem 3.47], we see that $\boldsymbol{h}_{i} \in \mathbf{H}^{\frac{1}{2}}(\Omega), i=1, \ldots, n$. Using [44, Lemma 10], we see that

$$
\mathbf{H}^{\frac{1}{2}}(\Omega) \hookrightarrow \hookrightarrow \mathbf{L}^{2}(\Omega) \cong \mathbf{L}^{2}(\Omega)^{*} \hookrightarrow \mathbf{H}_{0}^{-1}(\operatorname{curl}, \Omega) .
$$

Then, applying [40, Lemma 1.3.13], there exists a $\boldsymbol{H} \in C\left([0, T], \mathbf{L}^{2}(\Omega)\right)$ and a subsequence of $\boldsymbol{H}_{n}$ (denoted by the same symbol again) for which we have that

$$
\begin{cases}\boldsymbol{H}_{n} \rightarrow \boldsymbol{H} & \text { in } C\left([0, T], \mathbf{L}^{2}(\Omega)\right) \\ \partial_{t} \boldsymbol{H}_{n} \rightarrow \partial_{t} \boldsymbol{H} & \text { in } L^{2}\left([0, T], \mathbf{L}^{2}(\Omega)\right)\end{cases}
$$

Thanks to Lemma 4(iii), we have that $\int_{0}^{T}\left\|\partial_{t} \boldsymbol{H}_{n}\right\|^{2} \leq C$. Hence, $\overline{\boldsymbol{H}}_{n} \rightarrow \boldsymbol{H}$ in $L^{2}\left([0, T], \mathbf{L}^{2}(\Omega)\right)$.

iii. In this part of the proof, we apply Minty-Browder's trick [45, Chapter 9]. Due to the monotonicity, see Lemma 2, we can write that

$$
\int_{0}^{T}\left(\left|\nabla \times \overline{\boldsymbol{H}}_{n}\right|^{\beta-1} \nabla \times \overline{\boldsymbol{H}}_{n}-|\nabla \times \mathbf{u}|^{\beta-1} \nabla \times \mathbf{u}, \nabla \times \overline{\boldsymbol{H}}_{n}-\nabla \times \mathbf{u}\right) \geq 0,
$$


for all $\mathbf{u}$ with $\nabla \times \mathbf{u} \in L^{\beta+1}\left((0, T), \mathbf{L}^{\beta+1}(\Omega)\right)$. We want to take the limit $n \rightarrow \infty$ in (20). Because $\nabla \times \overline{\boldsymbol{H}}_{n} \rightarrow \nabla \times \boldsymbol{H} i n L^{\beta+1}\left((0, T), \mathbf{L}^{\beta+1}(\Omega)\right)$, we have that

$$
\lim _{n \rightarrow \infty} \int_{0}^{T}\left(|\nabla \times \mathbf{u}|^{\beta-1} \nabla \times \mathbf{u}, \nabla \times \overline{\boldsymbol{H}}_{n}-\nabla \times \mathbf{u}\right)=\int_{0}^{T}\left(|\nabla \times \mathbf{u}|^{\beta-1} \nabla \times \mathbf{u}, \nabla \times \boldsymbol{H}-\nabla \times \mathbf{u}\right) .
$$

From Lemma 4(iii), we get that $\left|\nabla \times \overline{\boldsymbol{H}}_{n}\right|^{\beta-1} \nabla \times \overline{\boldsymbol{H}}_{n} \in L^{\frac{\beta+1}{\beta}}\left((0, T), \mathbf{L}^{\frac{\beta+1}{\beta}}(\Omega)\right)$, which is a reflexive Banach space. Therefore, $\left|\nabla \times \overline{\boldsymbol{H}}_{n}\right|^{\beta-1} \nabla \times \overline{\boldsymbol{H}}_{n} \rightarrow \mathbf{z i n} L^{\frac{\beta+1}{\beta}}\left((0, T), \mathbf{L}^{\frac{\beta+1}{\beta}}(\Omega)\right)$, see [43, Theorem 1 p. 126]. Hence,

$$
\lim _{n \rightarrow \infty} \int_{0}^{T}\left(\left|\nabla \times \overline{\boldsymbol{H}}_{n}\right|^{\beta-1} \nabla \times \overline{\boldsymbol{H}}_{n}, \nabla \times \mathbf{u}\right)=\int_{0}^{T}(\mathbf{z}, \nabla \times \mathbf{u}) .
$$

Note that $\overline{\boldsymbol{F}}_{n} \rightarrow \boldsymbol{F}$ in $L^{2}\left([0, T], \mathbf{L}^{2}(\Omega)\right)$. Furthermore, we obtain due to (i) and (ii) that

$$
\begin{aligned}
\lim _{n \rightarrow \infty} g(\beta) \int_{0}^{T}\left(\left|\nabla \times \overline{\boldsymbol{H}}_{n}\right|^{\beta-1} \nabla \times \overline{\boldsymbol{H}}_{n}, \nabla \times \overline{\boldsymbol{H}}_{n}\right) \\
\stackrel{(19))}{=} \lim _{n \rightarrow \infty} \int_{0}^{T}\left[\left(\overline{\boldsymbol{F}}_{n}, \overline{\boldsymbol{H}}_{n}\right)-\left(\partial_{t} \boldsymbol{H}_{n}, \overline{\boldsymbol{H}}_{n}\right)-f(\beta)\left(\nabla \times \overline{\boldsymbol{H}}_{n}, \nabla \times \overline{\boldsymbol{H}}_{n}\right)\right. \\
\left.\quad-f(\beta)\left(\mathcal{K}_{0} \star \overline{\boldsymbol{H}}_{n}(t-\tau), \nabla \times \overline{\boldsymbol{H}}_{n}\right)\right] \\
\stackrel{(\star)}{\leq} \int_{0}^{T}\left[(\boldsymbol{F}, \boldsymbol{H})-\left(\partial_{t} \boldsymbol{H}, \boldsymbol{H}\right)-f(\beta)(\nabla \times \boldsymbol{H}, \nabla \times \boldsymbol{H})-f(\beta)\left(\mathcal{K}_{0} \star \boldsymbol{H}, \nabla \times \boldsymbol{H}\right)\right] \\
=\lim _{n \rightarrow \infty} \int_{0}^{T}\left[\left(\boldsymbol{F}_{n}, \boldsymbol{H}\right)-\left(\partial_{t} \boldsymbol{H}_{n}, \boldsymbol{H}\right)-f(\beta)\left(\nabla \times \overline{\boldsymbol{H}}_{n}, \nabla \times \boldsymbol{H}\right)\right. \\
\left.\quad-f(\beta)\left(\mathcal{K}_{0} \star \overline{\boldsymbol{H}}_{n}(t-\tau), \nabla \times \boldsymbol{H}\right)\right] \\
\stackrel{(19)}{=} \lim _{n \rightarrow \infty} g(\beta) \int_{0}^{T}\left(\left|\nabla \times \overline{\boldsymbol{H}}_{n}\right|^{\beta-1} \nabla \times \overline{\boldsymbol{H}}_{n}, \nabla \times \boldsymbol{H}\right) \\
=g(\beta) \int_{0}^{T}(\mathbf{z}, \nabla \times \boldsymbol{H}) .
\end{aligned}
$$

The inequality $(\star)$ is valid by the weak lower semicontinuity of the norm. Therefore, passing to the limit for $n \rightarrow \infty$ in (20), we get

$$
\int_{0}^{T}\left(\mathbf{z}-|\nabla \times \mathbf{u}|^{\beta-1} \nabla \times \mathbf{u}, \nabla \times \boldsymbol{H}-\nabla \times \mathbf{u}\right) \geq 0
$$

Now, we show that $\mathbf{z}=|\nabla \times \boldsymbol{H}|^{\beta-1} \nabla \times \boldsymbol{H}$. First, we put $\mathbf{u}=\boldsymbol{H}+\varepsilon \mathbf{v}$ for any $\mathbf{v}$ with $\nabla \times \mathbf{v} \in L^{\beta+1}\left((0, T), \mathbf{L}^{\beta+1}(\Omega)\right)$ and $\varepsilon>0$. Then, we get for (21) after dividing by $-\varepsilon$ that

$$
\int_{0}^{T}\left(\mathbf{z}-|\nabla \times(\boldsymbol{H}+\varepsilon \mathbf{v})|^{\beta-1} \nabla \times(\boldsymbol{H}+\varepsilon \mathbf{v}), \nabla \times \mathbf{v}\right) \leq 0
$$


Next, taking the limit $\varepsilon \rightarrow 0$, we get

$$
\int_{0}^{T}\left(\mathbf{z}-|\nabla \times \boldsymbol{H}|^{\beta-1} \nabla \times \boldsymbol{H}, \nabla \times \mathbf{v}\right) \leq 0 .
$$

The reverse inequality holds also true $(\mathbf{v} \leftrightarrow-\mathbf{v})$ and therefore,

$$
\int_{0}^{T}\left(\mathbf{z}-|\nabla \times \boldsymbol{H}|^{\beta-1} \nabla \times \boldsymbol{H}, \nabla \times \mathbf{v}\right)=0,
$$

for all $\mathbf{v}$ with $\nabla \times \mathbf{v} \in L^{\beta+1}\left((0, T), \mathbf{L}^{\beta+1}(\Omega)\right)$. From this, we conclude that $\mathbf{z}=|\nabla \times \boldsymbol{H}|^{\beta-1} \nabla \times \boldsymbol{H}$ a.e. in $Q_{T}$.

iv. Let us integrate (19) in time to get for any $t \in(0, T)$ and $\varphi \in \mathbf{V}_{0}$ that

$$
\begin{aligned}
& \int_{0}^{t}\left(\partial_{t} \boldsymbol{H}_{n}, \boldsymbol{\varphi}\right)+f(\beta) \int_{0}^{t}\left(\nabla \times \overline{\boldsymbol{H}}_{n}, \nabla \times \boldsymbol{\varphi}\right) \\
& \quad+g(\beta) \int_{0}^{t}\left(\left|\nabla \times \overline{\boldsymbol{H}}_{n}\right|^{\beta-1} \nabla \times \overline{\boldsymbol{H}}_{n}, \nabla \times \boldsymbol{\varphi}\right)=\int_{0}^{t}\left(\overline{\boldsymbol{F}}_{n}, \boldsymbol{\varphi}\right) \\
& \quad-f(\beta) \int_{0}^{t}\left(\mathcal{K}_{0} \star \overline{\boldsymbol{H}}_{n}(t-\tau), \nabla \times \boldsymbol{\varphi}\right) .
\end{aligned}
$$

We pass to the limit for $n \rightarrow \infty$. On the LHS, for the first term we use (ii), for the second term (i) and finally for the third term we apply (iii). For the RHS, we apply that $\overline{\boldsymbol{F}}_{n} \rightarrow$ $\boldsymbol{F}$ in $L^{2}\left([0, T], \mathbf{L}^{2}(\Omega)\right)$ and $(i i)$. We arrive at

$$
\begin{aligned}
& \int_{0}^{t}\left(\partial_{t} \boldsymbol{H}, \boldsymbol{\varphi}\right)+f(\beta) \int_{0}^{t}(\nabla \times \boldsymbol{H}, \nabla \times \boldsymbol{\varphi}) \\
& \quad+g(\beta) \int_{0}^{t}\left(|\nabla \times \boldsymbol{H}|^{\beta-1} \nabla \times \boldsymbol{H}, \nabla \times \boldsymbol{\varphi}\right)=\int_{0}^{t}(\boldsymbol{F}, \boldsymbol{\varphi})-f(\beta) \int_{0}^{t}\left(\mathcal{K}_{0} \star \boldsymbol{H}, \nabla \times \boldsymbol{\varphi}\right) .
\end{aligned}
$$

Finally, differentiating the resulting identity with respect to the time variable $t$, shows that $\boldsymbol{H}$ is a weak solution of (16). Up to now, we have only proven the convergence of the approximate solution only for a subsequence of $\overline{\boldsymbol{H}}_{n}$. But, if we take into account Theorem 1, we obtain the convergence of the whole sequence to the unique weak solution of (16) in corresponding spaces.

The following theorem addresses the error estimates for the time discretization.

Theorem 5 (Error). Suppose that $\boldsymbol{F} \in \operatorname{Lip}\left([0, T], \mathbf{L}^{2}(\Omega)\right)$. If $\boldsymbol{H}_{0} \in \mathbf{V}$ then

$$
\max _{t \in[0, T]}\left\|\boldsymbol{H}_{n}(t)-\boldsymbol{H}(t)\right\|^{2}+\int_{0}^{T}\left\|\nabla \times\left[\overline{\boldsymbol{H}}_{n}-\boldsymbol{H}\right]\right\|_{\mathbf{L}^{\beta+1}(\Omega)}^{\beta+1} \leq C \tau .
$$

Please note that the positive constant $C$ in this estimates is of the form $C e^{C T}$.

Proof. We subtract (16) from (19), set $\boldsymbol{\varphi}=\overline{\boldsymbol{H}}_{n}-\boldsymbol{H}$ and integrate in time to get

$$
\frac{1}{2}\left\|\boldsymbol{H}_{n}(t)-\boldsymbol{H}(t)\right\|^{2}+f(\beta) \int_{0}^{t}\left\|\nabla \times \overline{\boldsymbol{H}}_{n}-\nabla \times \boldsymbol{H}\right\|^{2}
$$




$$
\begin{aligned}
& +g(\beta) \int_{0}^{t}\left(\left|\nabla \times \overline{\boldsymbol{H}}_{n}\right|^{\beta-1} \nabla \times \overline{\boldsymbol{H}}_{n}-|\nabla \times \boldsymbol{H}|^{\beta-1} \nabla \times \boldsymbol{H}, \nabla \times\left(\overline{\boldsymbol{H}}_{n}-\boldsymbol{H}\right)\right) \\
& =\int_{0}^{t}\left(\overline{\boldsymbol{F}}_{n}-\boldsymbol{F}, \overline{\boldsymbol{H}}_{n}-\boldsymbol{H}\right)+\int_{0}^{t}\left(\partial_{t} \boldsymbol{H}_{n}-\partial_{t} \boldsymbol{H}, \boldsymbol{H}_{n}-\overline{\boldsymbol{H}}_{n}\right) \\
& +\int_{0}^{t}\left(\mathcal{K}_{0} \star\left[\overline{\boldsymbol{H}}_{n}(s-\tau)-\boldsymbol{H}(s)\right], \nabla \times\left[\overline{\boldsymbol{H}}_{n}(s)-\boldsymbol{H}(s)\right]\right) \mathrm{d} s .
\end{aligned}
$$

In the following estimates, we frequently use that

$$
\left\|\boldsymbol{H}_{n}(t)-\overline{\boldsymbol{H}}_{n}(t)\right\| \leq \tau\left\|\partial_{t} \boldsymbol{H}_{n}(t)\right\| \quad \text { for } t \in[0, T]
$$

The third term in the LHS can be bounded below by Lemma 2. The first term in the RHS can be estimated by using the Lipschitz continuity of $\boldsymbol{F}$, see [11, Theorem 4]. For the last term of (22), we calculate that

$$
\begin{aligned}
& \left|\int_{0}^{t}\left(\mathcal{K}_{0} \star\left[\overline{\boldsymbol{H}}_{n}(s-\tau)-\boldsymbol{H}(s)\right], \nabla \times\left[\overline{\boldsymbol{H}}_{n}(s)-\boldsymbol{H}(s)\right]\right) \mathrm{d} s\right| \\
& \quad \stackrel{(10)}{\leq} \varepsilon \int_{0}^{t}\left\|\nabla \times\left[\overline{\boldsymbol{H}}_{n}(s)-\boldsymbol{H}(s)\right]\right\|^{2} \mathrm{~d} s+C_{\varepsilon} \int_{0}^{t}\left\|\overline{\boldsymbol{H}}_{n}(s-\tau)-\boldsymbol{H}(s)\right\|^{2} \mathrm{~d} s \\
& \quad \leq \varepsilon \int_{0}^{t}\left\|\nabla \times\left[\overline{\boldsymbol{H}}_{n}(s)-\boldsymbol{H}(s)\right]\right\|^{2} \mathrm{~d} s+C_{\varepsilon} \int_{0}^{t}\left\|\boldsymbol{H}_{n}(s)-\boldsymbol{H}(s)\right\|^{2} \mathrm{~d} s+C_{\varepsilon} \tau^{2} .
\end{aligned}
$$

It remains to estimate the second term on the RHS in (22). Using Lemma 4(iii), we obtain

$$
\left|\int_{0}^{t}\left(\partial_{t} \boldsymbol{H}_{n}-\partial_{t} \boldsymbol{H}, \boldsymbol{H}_{n}-\overline{\boldsymbol{H}}_{n}\right)\right| \leq \sqrt{\int_{0}^{t}\left\|\partial_{t} \boldsymbol{H}_{n}-\partial_{t} \boldsymbol{H}\right\|^{2}} \sqrt{\int_{0}^{t}\left\|\boldsymbol{H}_{n}-\overline{\boldsymbol{H}}_{n}\right\|^{2}} \lesssim \tau
$$

Putting things together, choosing a sufficiently small positive $\varepsilon$ and applying Grönwall's argument, we conclude the proof.

\section{CONCLUSION}

In this contribution, a vectorial nonlocal nonlinear parabolic problem (8) in terms of the magnetic field for an intermediate state between type-I and type-II superconductivity is proposed and analyzed. This model is obtained from the eddy current version of the Maxwell equations, the two-fluid model of London and London, the nonlocal representation of the superconductive current by Eringen and the power law by Rhyner. A semi-implicit time-discrete scheme based on the backward Euler method is developed wherein the convolution is taken explicitly. The well-posedness of the problem is shown under low regularity assumptions and suboptimal error estimates are derived for the time-discretization.

$$
2014 .
$$

This paper arises from a lecture presented at the CMMSE 2014 Conference, Cadiz, Spain, July 


\section{References}

1. A. Alonso, A mathematical justification of the low-frequency heterogeneous time-harmonic maxwell equations, Math Models Methods Appl Sci 9 (1999), 475-489.

2. H. Ammari, A. Buffa, and J.-C Nédélec, A justification of eddy currents model for the maxwell equations, SIAM J Appl Math 60 (2000), 1805-1823.

3. S. J. Chapman, A hierarchy of models for type-II superconductors, SIAM Rev 42 (2000), 555-598.

4. S. J. Chapman, Macroscopic models of superconductivity, ICIAM 99, Proceedings of the 4th international congress on industrial \& applied mathematics, Edinburgh, GB, July 5-9, 1999, Oxford University Press, Oxford, 2000, pp 23-34.

5. S. J. Chapman and D. R. Heron, A hierarchy of models for superconducting thin films, SIAM J Appl Math 63 (2003), 2087-2127.

6. F. London and H. London, The electromagnetic equations of the supraconductor, Proc R Soc Lond Ser A 149 (1935), 71-88.

7. M. Fabrizio and A. Morro, Electromagnetism of continuous media, Oxford University Press, New York, 2003.

8. P. Monk, Finite element methods for Maxwell's equations, Oxford University Press, New York, 2003.

9. A. B. Pippard, An experimental and theoretical study of the relation between magnetic field and current in a superconductor, Proc R Soc Lond Ser A 216 (1953), 547-568.

10. A. C. Eringen, Electrodynamics of memory-dependent nonlocal elastic continua, J Math Phys 25 (1984), 3235-3249.

11. M. Slodička and K. Van Bockstal, A nonlocal parabolic model for type- $\{\mathrm{I}\}$ superconductors, Numerical Methods for Partial Differ Equ 30 (2014), 1821-1853.

12. K. Van Bockstal and M. Slodička, Error estimates for the full discretization of a nonlocal parabolic model for type-i superconductors, J Comput Appl Math 275 (2015), 516-526.

13. C. P. Bean, Magnetization of high-field superconductors, Rev Mod Phys 36 (1964), 31-39.

14. A. Bossavit, Numerical modelling of superconductors in three dimensions: a model and a finite element method, IEEE Trans Magn 30 (1994), 3363-3366.

15. G. Barnes, M. McCulloch, and D. Dew-Hughes, Computer modelling of type II superconductors in applications, Superconductor Sci Technol 12 (1999), 518.

16. J. W. Barrett and L. Prigozhin, Bean's critical-state model as the $p \rightarrow \infty$ limit of an evolutionary p-laplacian equation, Nonlinear Anal Theory Methods Appl 6 (2000), 977-993.

17. H.-M. Yin, B. Li, and J. Zou, A degenerate evolution system modeling bean's critical-state type-II superconductors, Discrete Continuous Dyn Syst 8 (2002), 781-794.

18. L. Prigozhin and V. Sokolovsky, Ac losses in type-II superconductors induced by nonuniform fluctuations of external magnetic field, IEEE Trans Appl Superconductivity 14 (2004), 69-81.

19. W. Wei and H.-M. Yin, Numerical solutions to bean's critical-state model for type-II superconductors, Int J Numer Anal Model 2 (2005), 479-488.

20. J. Rhyner, Magnetic properties and ac-losses of superconductors with power law current-voltage characteristics, Phys C 212 (1993), 292-300.

21. I. D. Mayergoyz, Nonlinear diffusion of electromagnetic fields: with applications to eddy currents and superconductivity, Electromagnetism, Elsevier Science, United States of America, 1998.

22. E. H. Brandt, Superconductors of finite thickness in a perpendicular magnetic field: strips and slabs, Phys Rev B 54 (1996), 4246-4264.

23. A. Gurevich and M. Friesen, Nonlinear transport current flow in superconductors with planar obstacles, Phys Rev B 62 (2000), 4004-4025. 
24. C. M. Elliott and Y. Kashima, On macroscopic critical state models of superconductivity in 3d. Proceedings of Czech-Japanese Seminar in Applied Mathematics 2005, Kuju, Japan, September 15-18, 2005, Kyushu University, Fukuoka, The 21st Century COE Program “DMHF,” 2006, pp. 39-55.

25. C. M. Elliott and Y. Kashima, A finite-element analysis of critical-state models for type-II superconductivity in 3d, IMA J Numer Anal 27 (2007), 293-331.

26. M. Slodička and E. Janiková, Convergence of the backward euler method for type-II superconductors, J Math Anal Appl 342 (2008), 1026-1037.

27. E. Babaev and M. Speight, Semi-meissner state and neither type-I nor type-II superconductivity in multicomponent superconductors, Phys Rev B 72 (2005), 180502.

28. J. Nagamatsu, N. Nakagawa, T. Muranaka, Y. Zenitani, and J. Akimitsu, Superconductivity at 39K in magnesium diboride, Nature 410 (2001), 63-64.

29. V. Moshchalkov, M. Menghini, T. Nishio, Q. H. Chen, A. V. Silhanek, V. H. Dao, L. F. Chibotaru, N. D. Zhigadlo, and J. Karpinski, Type-1.5 superconductivity, Phys Rev Lett 102 (2009), 117001.

30. T. Nishio, V. H. Dao, Q. Chen, L. F. Chibotaru, K. Kadowaki, and V. V. Moshchalkov, Scanning squid microscopy of vortex clusters in multiband superconductors, Phys Rev B 81 (2010), 020506.

31. J. Carlström, E. Babaev, and M. Speight, Type-1.5 superconductivity in multiband systems: effects of interband couplings, Phys Rev B 83 (2011), 174509.

32. M. Silaev and E. Babaev, Microscopic theory of type-1.5 superconductivity in multiband systems, Phys Rev B 84 (2011), 094515.

33. E. Babaev, J. Carlström, J. Garaud, M. Silaev, and J. M. Speight, Type-1.5 superconductivity in multiband systems: magnetic response, broken symmetries and microscopic theory-a brief overview, Phys C 479 (2012), 2-14.

34. C. N. Varney, K. A. H. Sellin, Q.-Z. Wang, H. Fangohr, and E. Babaev, Hierarchical structure formation in layered superconducting systems with multi-scale inter-vortex interactions, J Phys: Condens Matter 25 (2013), 415702.

35. J. Carlström, J. Garaud, and E. Babaev, Semi-meissner state and nonpairwise intervortex interactions in type-1.5 superconductors, Phys Rev B 84 (2011), 134515.

36. M. Silaev and E. Babaev, Microscopic derivation of two-component ginzburg-landau model and conditions of its applicability in two-band systems, Phys Rev B 85 (2012), 134514.

37. R. B. Guenther and J. W. Lee, Partial differential equations of mathematical physics and integral equations, Dover books on mathematics, Dover Publications, United States of America, 1988.

38. M. Slodička, Nonlinear diffusion in type-II superconductors, J Comput Appl Math 215 (2008), 568-576.

39. E. DiBenedetto, Degenerate Parabolic Equations, Springer-Verlag, New York, 1993.

40. J. Kačur, Method of Rothe in evolution equations, volume 80 of Teubner Texte zur Mathematik. Teubner, Leipzig, 1985.

41. M. M. Vainberg, Variational method and method of monotone operators in the theory of nonlinear equations, Translated from Russian by A. Libin, Translation edited by D. Louvish. A Halsted Press Book. New York-Toronto: John Wiley \& Sons; Jerusalem, London: Israel Program for Scientific Translations. xi, 1973, 356 p.

42. M. Slodička and V. Zemanová, Time-discretization scheme for quasi-static maxwell's equations with a non-linear boundary condition, J Comput Appl Math 216 (2008), 514-522.

43. K. Yosida, Functional analysis, Classics in Mathematics, World Publishing Company, New York, 1980.

44. G. Palatucci, O. Savin, and E. Valdinoci, Local and global minimizers for a variational energy involving a fractional norm, Ann Mat Pura Appl 192 (2013), 673-718.

45. L. C. Evans, Partial differential equations, volume 19 of Graduate Studies in Mathematics. American Mathematical Society, Providence, RI, 1998. 